Cahiers $d u$ MONDE RUSSE

\section{Cahiers du monde russe}

Russie - Empire russe - Union soviétique et États indépendants

$49 / 4 \mid 2008$

Destins individuels et terreur. Jeunesse dans la société post-stalinienne

\title{
Mark D. Steinberg, Heather J. Coleman (eds), Sacred stories
}

\section{Aleksandr Lavrov}

\section{OpenEdition}

Journals

Édition électronique

URL : https://journals.openedition.org/monderusse/7003

DOI : 10.4000/monderusse. 7003

ISSN : $1777-5388$

Éditeur

Éditions de l'EHESS

Édition imprimée

Date de publication : 28 décembre 2008

Pagination : 826-830

ISBN : 978-2-7132-2197-2

ISSN : $1252-6576$

Référence électronique

Aleksandr Lavrov, « Mark D. Steinberg, Heather J. Coleman (eds), Sacred stories », Cahiers du monde russe [En ligne], 49/4 | 2008, mis en ligne le 24 décembre 2009, consulté le 03 septembre 2022. URL http://journals.openedition.org/monderusse/7003; DOI : https://doi.org/10.4000/monderusse.7003

Ce document a été généré automatiquement le 3 septembre 2022

Tous droits réservés 


\title{
Mark D. Steinberg, Heather J. Coleman (eds), Sacred stories
}

\author{
Aleksandr Lavrov
}

\section{RÉFÉRENCE}

Mark D. STEINBERG, Heather J. COLEMAN (eds), Sacred stories. Religion and Spirituality in Modern Russia. Bloomington, Indianopolis : Indiana University Press, 2007, 420 p.

1 Le recueil d'articles édité par Mark D. Steinberg et Heather J. Coleman présente deux particularités. D'abord il ne s'agit pas d'une collection d'études sur l'orthodoxie russe : l'ensemble des religions et des croyances présentes dans l'Empire russe du XVIII ${ }^{\mathrm{e}}$ au début $\mathrm{du} \mathrm{xx}^{\mathrm{e}}$ siècle sont abordées. Cela étant, on peut déplorer que la majorité des auteurs n'ait pas cherché à comparer des phénomènes provenant de deux régions différentes de l'Empire : ils ont préféré rester chez eux. Ensuite, les éditeurs ont introduit l'expression « histoires sacrées » dans le titre du recueil, dans la mesure où leur objectif principal est l'étude de textes narratifs. Dans l'introduction, ils expliquent que « la religion est comprise ici moins comme une histoire des institutions et des croyances arrêtées que comme un terrain essentiel de l'imaginaire et de la pratique sociale, où l'ensemble des expériences quotidiennes ou extraordinaires, des idées, des croyances, et des émotions aide les hommes à comprendre leur vie ». Les « histoires sacrées », par conséquent, nous parlent « du pouvoir et de la résistance, de la communauté et de l'individualité, de la sphère publique et de la vie privée, de classe et de genre, et, avant tout, de modernité » (p. 1).

2 Pour étayer leur point de vue, les auteurs se réfèrent à la traduction américaine des Formes élémentaires de la vie religieuse d'Émile Durkheim (1912) - dont ils reprennent l'opposition entre "sacré» et "profane» ainsi que la notion de "communauté morale »-, et à Foi et Savoir de Jacques Derrida (1996), cité, là aussi, dans la traduction américaine. À Derrida, les auteurs empruntent la distinction entre « l'expérience de la sacralité » et l'engagement pour une foi. En revanche, les grands noms de la sociologie 
américaine de la religion (y compris Peter Berger) sont absents du texte d'introduction et exilés dans la bibliographie. Il me semble que ce choix de références, assez conventionnel, ne reflète pas les préférences personnelles des deux éditeurs, mais résulte de la difficulté qu'il y a à réunir sous un même toit des auteurs dont les approches sont si clairement différentes. Par exemple, Steinberg et Coleman sont plutôt réservés à l'égard de la « culture populaire » si controversée (p. 7-8), alors qu'on trouve dans le recueil non seulement un article de Gregory Freeze, qui oppose "orthodoxie populaire» et " officielle", mais aussi celui de Vera Shevzov qui insiste sur la "piété populaire" (p. 7-8, 147).

On peut essayer de classer les articles en deux catégories : dans la première figurent les textes correspondant pleinement au titre du recueil, qui sont directement consacrés par conséquent à l'analyse de textes narratifs religieux ou de discours sur la religion, dans la seconde ceux qui appartiennent à l'histoire des idées ou à l'histoire littéraire.

Un des plus intéressants du premier groupe est, sans conteste, l'article de Nadieszda Kizenko "Confessions écrites et construction de la narration religieuse ». Il porte sur une source absolument inattendue - en l'occurrence les confessions écrites de fidèles, envoyées à l'archiprêtre Ioann Sergiev (Ioann de Kronštadt) et conservées dans ses archives privées $^{1}$. Dans l'esprit de la discipline pénitentielle orthodoxe, un confesseur ayant accepté une confession sous forme écrite aurait dû détruire le document après l'absolution des péchés. Ioann de Kronštadt a évidemment négligé cette dernière précaution. Kizenko étudie à cette source unique à la fois du point de vue statistique et du point de vue du discours. Bien que les femmes prédominent parmi les auteurs des lettres (121 pour 34 hommes), Kizenko y voit plutôt le reflet de l'entourage de Ioann de Kronštadt plutôt qu'une "féminisation de la piété » (p. 97). Plusieurs documents sont étudiés de très près - par exemple, la confession d'un représentant «repenti » de l'intelligentsia, qui souligne l'influence pernicieuse des idées de Léon Tolstoï. Partant de là, Kizenko analyse avec précision la genèse de l'image de Tolstoï et des tolstoïens chez Ioann de Kronštadt et explique ainsi son attitude intransigeante à l'égard du grand écrivain (p. 98-99).

Gregory Freeze aborde son sujet selon sa méthode habituelle - l'analyse d'une série de documents d'archives, réunis par un sujet commun (ici, des demandes de divorce, provenant des archives du diocèse orthodoxe de Lituanie). Il distingue les récits émanant de privilégiés, rédigés souvent avec l'aide d'un avocat, des récits de non-privilégiés, révélant une ignorance totale de la législation en vigueur (on inventait par exemple des causes de divorce qui n'étaient pas reconnues par la loi). Bien entendu, le récit masculin, qui se réfère surtout au pouvoir patriarcal de l'époux, diffère du récit féminin, qui évoque un mariage idéal, caractérisé par le partenariat, la réciprocité et l'amour mutuel. L'auteur compare aussi les récits profanes aux religieux, tout en tenant compte du caractère multiconfessionnel du diocèse ${ }^{2}$. Christine Worobec analyse les listes de pèlerins guéris, qui furent tenues à jour pour quatre saints orthodoxes (Dimitrij de Rostov, Pitirim de Tambov, Ioasaf de Belgorod et Serafim de Sarov). Elle réunit dans son analyse les ingrédients typiques du médiéviste: le classement des miraculés selon le sexe, l'origine géographique et le groupe social, avec une analyse du discours des malades guéris comparé au discours médical. Elle peut ainsi identifier les cultes à portée nationale (Serafim de Sarov) et ceux qui n'ont qu'une diffusion locale (par exemple, Pitirim de Tambov). On discerne également les cultes où les pèlerins-paysans dominent (Dimitrij de Rostov et Serafim de Sarov) (p. 24-25, 29)3. Les femmes forment $50 \%$ des 
guéris, ce qui permet à Worobec d'affirmer qu'aucune «féminisation de la piété » à l'époque moderne n'est perceptible (p. 30).

6 Contrairement aux précédents, l'article de Roy Robson (« Les Solovki se transforment : récits de pèlerins, modernisation et vie monastique à la fin de la période impériale»), consacré lui aussi aux pèlerinages, ne semble pas convaincant, tantôt à cause du choix des sources (récits de pèlerins publiés), tantôt à cause de leur exploitation, tantôt enfin parce que l'auteur oublie la longue durée. Il affirme que la métamorphose du monastère des Solovki « de cloître en véritable Mecque, capable de loger et de nourrir des milliers de pèlerins à la fois » a eu lieu "à la fin de la période impériale » et qu'elle était « l'un des symptômes révélateurs d'un changement profond de la religion populaire » à cette époque. En réalité, les Solovki faisaient partie des sites monastiques les plus visités par les pèlerins déjà pendant la période moscovite. Aussi les nouveaux moyens de transport, en augmentant le nombre des pèlerins, n'ont-ils rien changé au caractère panrusse du pèlerinage.

7 Trois autres articles - par Heather J.Coleman, par Paul W. Werth et par Nicholas Breyfogle - sont unis par un même sujet, la situation des minorités religieuses. Coleman ("Les récits de violence contre les dissidents religieux dans un village orthodoxe») montre qu'en abolissant certaines restrictions des droits qui s'appliquaient aux représentants de minorités religieuses, on n'améliorait pas immédiatement leur situation réelle. Cette émancipation fut en effet accompagnée de nombreuses agressions d'orthodoxes contre les prétendus « sectaires » (en premier lieu contre les baptistes). Ces violences sont assez rarement évoquées dans l'historiographie. L'auteur analyse plusieurs discours consacrés au problème - celui de la majorité orthodoxe, dont les motions d'assemblées villageoises décidant d'expulser les baptistes, mais aussi le discours des représentants des minorités elles-mêmes, celui de la presse d'opposition qui, voulant à tout prix défendre l'innocence du "peuple", rendait entièrement responsable des agressions le clergé orthodoxe. Quant à la presse ecclésiastique orthodoxe, elle brodait sur le thème des orthodoxes abandonnés par les autorités, qui n'avaient d'autre choix que de se protéger eux-mêmes. L'auteur évoque deux détails révélateurs de l'acharnement des agresseurs - la comparaison avec le pogrom, proposée par le journal libéral Reč, et la rumeur persistante de "rituels sanglants ", répandue pour susciter des agressions contre les prétendus « sectaires » (p. 212, 209).

Paul Werth (« Arbitres de la liberté de conscience : l'État, la religion et le problème de la conversion d'une confession à l'autre après $1905 »)$ étudie le discours des hauts fonctionnaires de l'ancien régime russe, qui essayaient à tout prix d'interpréter les normes de l'édit du 17 avril 1905 sur la tolérance religieuse de la manière la plus restrictive. L'édit, qui permettait à un orthodoxe de se convertir à une autre confession chrétienne, entendait laisser aux gréco-catholiques la possibilité de quitter l'Église russe orthodoxe et de rejoindre leur Église. Mais, la procédure de sortie de l'Église russe n'ayant pas été précisée, les autorités continuaient de traiter comme orthodoxes un grand groupe de croyants, qui appartenaient en réalité à l'Église gréco-catholique. Plus difficile encore était le cas des orthodoxes qui voulaient se convertir à une religion non chrétienne, par exemple, celui des Maris christianisés qui voulaient revenir à leurs croyances préchrétiennes. En effet, les bureaucrates d'ancien régime pouvaient accepter un « retour » à la religion des ancêtres, mais en aucun cas un libre choix de l'identité religieuse. Le statut des juifs, victimes de discriminations à plusieurs niveaux (dont la zone de résidence), n'était pas moins complexe. Les autorités voulaient conserver la 
situation d'avant 1905, dans laquelle la conversion d'un juif à une confession chrétienne entraînait son émancipation juridique. En revanche, la conversion d'un juif après 1905 à une prétendue «secte» ou à une religion non chrétienne (par exemple, à l'islam), ne devait pas améliorer la situation juridique du converti, qui restait soumis à toutes les discriminations imposées aux juifs. Ainsi se formait un discours très particulier dans lequel le fait d'être « juif » ne renvoyait pas seulement à l'identité religieuse, mais aussi aux origines d'un individu.

Nicholas B. Breyfogle a publié récemment une monographie sur les dissidents religieux russes, mal vus des autorités, mais utilisés malgré cela comme colons en Transcaucasie au milieu de populations qui étaient encore plus suspectes qu'eux-mêmes ${ }^{4}$. Dans son article il pose la question, essentielle, de la formation d'un « espace public » dans la Russie d'ancien régime, mais son sujet - la construction d'une maison de prière des molokanes à Bakou - reste trop limité pour servir de base à des généralisations (« Prière et politique de l'espace : la construction de bâtiments du culte pour les molokanes, la législation tsariste et la recherche d'un espace public dans la Russie impériale tardive »).

Deux articles consacrés à la vie religieuse juive sont particulièrement importants, dans la mesure où ils l'inscrivent dans le contexte de l'Empire russe. Sarah Abrevaya Stern pose le problème de la laïcisation à partir du premier quotidien en yiddish publié dans l'Empire russe, Der fraynd ("À la rencontre du siècle : la presse populaire yiddish»). Gabrielle Safran étudie les transformations de la légende hassidique où le rabbin convoque Dieu devant le tribunal pour avoir permis les souffrances du peuple juif. Cette histoire fut adaptée et réécrite par le folkloriste N. An-skij (S.-Z. Rappoport), deux fois en russe et deux fois en yiddish, la faisant entrer ainsi dans les deux littératures («Les rabbins révolutionnaires : la légende hassidique et le héros de la parole»).

11 Le deuxième groupe d'articles, tourné vers l'histoire des idées et l'histoire littéraire, comprend une étude de Marc D. Steinberg sur le langage des poètes-ouvriers russes au commencement $\mathrm{du} \mathrm{xx}^{\mathrm{e}}$ siècle $:$ il identifie un certain nombre de connotations religieuses (" "Le chemin de croix": blessures de l'esprit et pérégrinations des poètes ouvriers »). Alexei Kurbanovsky, au prix d'un travail méticuleux, retrouve les sources des textes écrits par Malevič, où il détecte les influences de Nietzsche, d'Henri Bergson et de Pavel Florenskij ( Les signes mystiques de Malevič: de l'iconoclasme à la nouvelle théologie»). Paul Valliere donne un article sur un sujet apparemment de sa propre invention: la "théologie de la culture» ("La théologie de la culture dans la Russie impériale tardive »). Bernice Glatzer Rosenthal essaie de montrer l'influence exercée par Nietzsche sur Dmitrij Merežkovskij, Vjačeslav Ivanov et Pavel Florenskij (« La nouvelle spiritualité: la confluence de l'orthodoxie et de Nietzsche dans la pensée religieuse russe »). Mais la tendance de l'auteur à traiter de la pensée russe et de l'orthodoxie " en

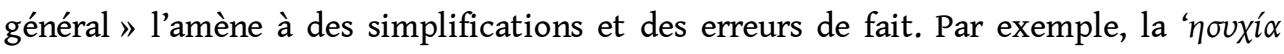
(" silence », « calme », " solitude »; d'où " hésychasme ») est translittérée de telle façon ( "Heschia », p. 335) que le mot devient méconnaissable ; les autres mots grecs subissent un traitement analogue. Le père-fondateur des "non-possesseurs » s'appelait Nil de la Sora, et non pas " Nils ", comme l'auteur l'affirme à deux reprises (p. 335-336). Le synode de 1503 ne "réprima» pas les non-possesseurs, il consacra seulement l'échec de la tentative du grand-prince de séculariser les possessions de l'Église - tentative soutenue précisément par les «non-possesseurs ». Il n'est pas de tradition plus louable que celle des publications anglophones, et spécialement américaines, qui consiste à faire relire les 
papers par ses collègues. Elle permet en temps ordinaire d'étouffer de semblables erreurs dans l'œuf, c'est-à-dire au niveau du manuscrit. Manifestement ce n'est pas le cas ici.

Si l'on peut se permettre une remarque au sujet du recueil tout entier, on doit faire observer que dans la bibliographie des auteurs, les titres américains prédominent, tandis que les publications en russe, en français ou en allemand sont nettement minoritaires. Ce reproche peut être adressé même aux meilleurs articles du recueil, comme par exemple à celui de Vera Shevzov (« Scripting the Gaze: la liturgie, les homélies, et l'icône de la Mère de Dieu de Kazan' à la fin de l'Empire russe »). En retraçant l'histoire du culte de l'icône de la Sainte Vierge de Kazan', l'auteur ignore l'étude fondamentale d'Anton Ebbinghaus, tout comme les travaux des chercheurs russes, consacrés au « Récit de l'invention... » de cette icône ${ }^{5}$.

\section{NOTES}

1. Nadieszda Kizenko lui a consacré une monographie, A Prodigal Saint: Father John of Kronstadt and the Russian People, University Park, 2000.

2. Freeze rapporte une histoire qui n'a rien de «sacré ", mais très caractéristique de Nicolas II. Un orthodoxe poursuit sa femme et l'amant de celle-ci, fils d'un banquer juif. Les ayant trouvés ensemble dans un hôtel, le mari abat son rival d'un coup de revolver et il est arrêté. Bien que le meurtre ait été prémédité, l'empereur - «sous l'influence peut-être de l'antisémitisme sousjacent ", écrit Freeze - fait usage de son droit de grâce et réduit la peine à trois mois de détention dans une prison militaire (p. 171).

3. La présence de Dimitrij de Rostov permet d'établir la comparaison entre le milieu du $\mathrm{XVIII}^{\mathrm{e}}$ siècle (ses guérisons ont eu lieu entre 1753 et 1762 ) et la deuxième moitié $\mathrm{du} \mathrm{XIX}^{\mathrm{e}}$ et le début du $\mathrm{Xx}^{\mathrm{e}}$ siècle (guérisons des trois autres saints).

4. Nicholas B. Breyfogle, Heretics and Colonizers: Forging Russia's Empire in the South Caucasus, Ithaca: Cornell University Press, 2005.

5. Anton Ebbinghaus, Die altrussischen Marienikonen-Legenden, Wiesbaden, 1991 ; D. M. Bulanin, "Skazanie ob ikone Bogomateri Kazanskoj Jaroslavskoj », in Slovar' knižnikov i knižnosti Drevnej Rusi, SPb., III/4, p.570-572; E. K. Romodanovskaja, «Skazanie o javlenii Kazanskoj ikony Bogorodicy v Tobol'ske ", in Hristianstvo i cerkov'v Rossii feodal'nogo perioda, Novosibirsk, 1988, p. 45-58 ; A. A. Romanova, « K istorii spiskov ikony Kazanskoj Bogomateri XVII v. : Skazanie ob ikone Bogomateri Kazanskoj Nižnelomovskoj », in Issledovanija po istorii srednevekovoj Rusi. K 80letiju Jurija Georgieviča Alekseeva, M., 2006, p. 468-480. 